\title{
Derivation, Comprehensive Analysis of Cancer- associated Fibroblasts and Their Correlation to Prognosis, Immune Status and Drug Sensitivity in Patients of Soft Tissue Sarcoma
}

\section{Bo Xiao}

the Second Xiangya Hospital of Central South University

Liyan Liu

the Second Xiangya Hospital of Central South University

Zhuoyuan Chen

the Second Xiangya Hospital of Central South University

Aoyu Li

the Second Xiangya Hospital of Central South University

Yu Xia

the Second Xiangya Hospital of Central South University

Pingxiao Wang

the Second Xiangya Hospital of Central South University

Ziyue Zhao

the Second Xiangya Hospital of Central South University

Cheng Xiang

the Second Xiangya Hospital of Central South University

Yi Zeng

the Second Xiangya Hospital of Central South University

Hui Li ( $\boldsymbol{\nabla}$ lihuix@csu.edu.cn )

the Second Xiangya Hospital of Central South University

Tao Xiao

the Second Xiangya Hospital of Central South University

\section{Research Article}

Keywords: soft-tissue sarcomas, CAFs, tumor microenvironment, prognostic, signature

Posted Date: February 2nd, 2022

DOI: https://doi.org/10.21203/rs.3.rs-1278219/v1 
License: (c) (i) This work is licensed under a Creative Commons Attribution 4.0 International License. Read Full License 


\section{Abstract}

Background: Soft tissue sarcoma is among the most challenging malignancies for clinicians and associated with poor prognosis. Former studies have revealed that cancer-associated fibroblasts (CAFs) are involved in tumor development, migration, invasion and metastasis.

Methods: DEGs and WGCNA were employed to obtain significant CAFs-related genes for constructing the signature. Somatic mutation, immune status, immune checkpoints and chemotherapy sensitivity were applied to define the characteristics of the identified signature. Differentially expressed genes between the distinct groups and WGCNA were employed to obtain CAFs score of each patient.

Results: 35 genes were identified as CAFs-related genes in soft tissue sarcoma samples. Then, 12 CAFsrelated genes were associated with overall survival in univariate Cox regression analysis and K-M method. The five genes signature was constructed based on the result from the LASSO regression analysis in train cohort. K-M curve showed that patients in the low CAFs score group had better survival, with immune infiltration and function significantly increased $(P<0.05)$. The $1-, 3$ - and 5- year survival AUC values were $0.773,0.724$, and 0.726 , respectively. The analysis of tumor mutations revealed that TP53, ATRX, TTN, RB1 and MUC16 were enriched in both clusters. The analysis of chemotherapy response demonstrated that most of chemotherapeutic drugs in GDSC database had lower IC50 in the high-risk group. All of the results were validated in test and whole cohorts.

Conclusion: This study strongly revealed the indispensable role of CAFs in soft tissue sarcoma and constructed the prognostic signature including five CAFs-related genes.

\section{Introduction}

Soft-tissue sarcomas (STS) are a family of rare and heterogeneous mesenchymal malignancies accounted for $1 \%$ of tumors in all adult ${ }^{1}$. In 2019 , approximate 13500 people were diagnosed with a soft tissue sarcoma in the US ${ }^{2}$. STS compasses more than 100 different histologic and molecular subtypes, and each subtype showing different clinical behavior ${ }^{2}$. With the development of modern genetic sequencing techniques, even the same subtype was also identified remarkable genetic heterogeneity. Despite this heterogeneity, the treatment standard for STS has remained surgery and adjuvant management ${ }^{3}$. However, merely $50 \% \sim 60 \%$ of patients with soft tissue sarcoma exhibit the survival rate of 5 -year whereas approximately half of the patients with distant metastasis ultimately succumb to the disease $\mathrm{e}^{4}$. Therefore, Comprehensive analysis the molecular characteristic of soft tissue sarcoma would advance the treatment and prognosis of soft tissue sarcoma.

Cancer-associated fibroblasts (CAFs) are a vital part of tumor microenvironment with multiple functions, which associated with tumor proliferation, angiogenesis, invasion, migration, chemoresistance, infiltration of immune cells and immunosuppression ${ }^{5}$. CAFs interact with immune components via secreting different factors, for example, collagen, chemokines, cytokine and Matrix Metalloproteinases (MMPs) ${ }^{6}$. 
CAFs mediate cancer cell proliferation and metastasis by secreting various factors, such as stromal cellderived factor 1 (SDF-1/CXCL12) and fibroblast growth factor-2 (FGF-2). CAFs regulate the recruitment and polarization of immune cells and angiogenesis in a pro-tumorigenic manner via secreting interleukins and chemokines (CXCL12) ${ }^{7,8}$. CAFs can avoid immune cell infiltration in tumors via constructing extracellular matrix (ECM) protein networks ${ }^{9}$. Besides, the enrichment of a-SMA+ CAFs in tumor microenvironment is associated with worse prognosis in numerous cancers ${ }^{10,11}$. Therefore, it is necessary to explore the underlying mechanism of cancer-associated fibroblasts (CAFs) related genes in soft tissue sarcoma.

As a systems biology method, bioinformatics is widely applied in cancer research. Through RNA sequencing, the underlying pathological mechanisms of diseases (including cancers) can be indicated via comparing the expression profile of different genes. TIMER2.0 (http://timer.cistrome.org) is an interactive web server for comprehensive analyzing the abundance of immune cells infiltration in cancers $^{12}$. Thus, the CAFs score of $x$ Cell in TIMER2.0 was used in this study. Besides, Weighted gene coexpression network analysis (WGCNA) was applied to obtain significant CAFs-related gene modules in soft tissue sarcomas samples. Therefore, we analyzed the prognosis value, tumor mutation, chemotherapy sensitivity of CAFs-related genes in soft tissue sarcoma patients.

\section{Methods}

\section{Patient inclusion}

The RNA-seq (FPKM) information and simple nucleotide variation of 265 samples (263 soft tissues sarcoma tissues and 2 adjacent normal tissues) and corresponding clinicopathological data were collected from The Cancer Genome Atlas (TCGA) (https://portal.gdc.cancer.gov) database. These tissue samples represented different subtypes: fibromatous neoplasms $(n=40)$, lipomatous neoplasms $(n=59)$, myomatous neoplasms $(n=105)$, nerve sheath tumors $(n=10)$, soft tissue tumors and sarcomas, NOS $(n=36)$ as well as synovial-like neoplasms $(n=10)$. The $x$ Cell algorithm that provided by TIMER2.0 (http://timer.cistrome.org) was employed to compute the CAFs score of soft tissues sarcoma patients. The workflow was shown in Figure 1. All data in this study were downloaded from public databases, and the authors were not involved in any animal or human experiments. Therefore, no additional ethical approval was required for this study.

\section{Identification of differentially expressed genes (DEGs)}

Based on the medium value of the CAFs score, the SARC cohort was divided into two subgroups to identify the DEGs. Then, the "limma" package (versions R 4.0.1) was applied to screen differential gene expression between these two subgroups. Genes with FDR< 0.05 were considered to be significantly differentially expressed.

\section{WGCNA identifying macrophage-specific genes}


We performed WGCNA by employing the "WGCNA" package in versions R 4.0.1. The gene significance was used to quantify the association between subgroups and individual genes. Similarly, the module membership was applied to show the relationship between module eigengenes and the profile of gene expression. CAFs-related genes were selected from the most significant modules associated with highCAFs. A power of beta $=5$ and a scale-free $\mathrm{R} 2=0.90$ were automatically computed by pickSoftThreshold and softConnectivity function, respectively, were regarded as softthreshold parameters to guarantee a scale-free topology network and generate a TOM matrix. After recomputing module eigengenes, the module dendrogram was applied to describe the relationship between the eigengenes and the subgroups through plotEigengeneNetworks function. The verboseScatterplot was used to visualize the relationship between module membership and gene significance. The genes that intersected between DEGs and CAFsrelated genes that identified by WGCNA were defined as Hub genes.

\section{Identification of a macrophages-specific gene expression signature}

Firstly, we then randomly divided the soft tissue sarcoma samples into the train and test cohorts using the "caret" package (versions $\mathrm{R}$ 4.0.1). Then, univariate Cox regression analysis and K-M survival method were employed to identify the correlation of Hub genes with the soft tissue sarcoma patients' overall survival by using the "survival" package (versions R 4.0.1) $(P<0.05)$. Subsequently, the CAFs-related gene signature was constructed via the least absolute shrinkage operator (LASSO) regression analysis in the $R$ "glmnet" package (versions R 4.0.1) to predict the soft tissue sarcoma patients' prognosis. The CAFs score of each sample was obtained by the following formula: CAFs score $=\sum$ icoefficient (gene $i$ ) $\times$ expression (gene $i$ ). The samples were classified as two subgroups in view of the median CAFs score. The Kaplan-Meier (K-M) method was applied to explore the overall survival of the soft tissue sarcoma patients in two groups via log-rank test utilizing the "survival" package (versions R 4.0.1). The timedependent receiver-operating characteristic (ROC) was used to check the prognostic capability of the CAFs signature utilizing the "survivalROC" package (versions R 4.0.1). Univariate and multivariate Cox regression analyses were employed based on the CAFs score and the available clinical information in the SARC cohort to verify the prognostic effect of CAFs signature.

\section{Estimation of TMB value and immune infiltration analysis}

Tumor mutation burden (TMB) was defined as the total number of somatic gene coding errors, base substitutions, insertions or deletions detected per megabyte bases of tumor tissue ${ }^{13}$. We calculated the total mutation frequency/the length of the human exon $(38 \mathrm{Mb})$ for every patient through Perl scripts ${ }^{14}$. Furthermore, all synonymous mutations were excluded for calculating TMB value. The 16 immune infiltrating cells and 13 immune related functions were quantified via SSGSEA in district risk subgroups of train cohort through using the "GSVA" and "GSEABase" package (versions R 4.0.1).

\section{Predicting chemotherapeutic response}

The Genomics of Drug Sensitivity in Cancer (GDSC) database was applied to predict the chemotherapeutic response for every soft tissue sarcoma patient. Cisplatin, docetaxel, doxorubicin, 
etoposide and methotrexate, which are common chemotherapy drugs for treating soft tissue sarcoma were selected for predicting the chemotherapeutic response. The R package "pRRophetic" (versions R 4.0.1) was used to conduct the prediction procedure, by which the half-maximal inhibitory concentration (IC50) of samples was predicted through ridge regression. The precision was computed via 10 -fold crossvalidation on the foundation of the GDSC training dataset ${ }^{15}$.

\section{Results}

\section{Identification of CAFs as a potential prognostic biomarker}

We first obtained the score of CAFs in the TCGA-SARC cohort utilizing the xCell algorithm. The K-M survival analysis indicated that the abundance of CAFs had a significant role on the overall survival of patients (Figure 2A). Patients with higher CAFs score had worse survival $(P=0.014)$. To further identify the genes associated with CAFs infiltration in the TCGA-SARC cohort, we divided the TCGA-SARC cohort into two subgroups based on the median value of CAFs score to screen the differentially expressed genes (DEGs). Then, a total of 185 genes were DEGs between the high and low CAFs groups in the SARC cohort. The heatmap demonstrates all DEGs in Figure 2B. Next, we used WGCNA to identify the module that had the most notable correlation with the abundance of CAFs in the TCGA-SARC cohort (Figure 2C). The greenyellow module indicated the most important association with a correlation level of 0.39 , while the association between module membership and gene significance was 0.36 (Figure 2D). Then, we draw the Venn diagram and obtained 35 hub genes that intersected between DEGs and genes in the most associated module (Figure 2E).

\section{Construction a CAFs-related gene expression signature}

Firstly, we merge the expression prolife of hub genes with survival information of SARC patients. Then, we employed the univariate Cox regression analysis and K-M survival analysis to assess the 35 hub genes. We found $20 \mathrm{CAFs}$-related genes was associated with overall survival of soft tissue sarcoma patients $(P<0.05)$ using the "survival" package (Figure $3 A)$. Besides, we identified 12 CAFs-related genes were also associated with overall survival in $\mathrm{K}-\mathrm{M}$ method $(\mathrm{P}<0.05)$ (Supplementary Figure $\mathrm{S} 1$ ).

Subsequently, the $256 \mathrm{soft}$ tissue sarcoma patients were randomly assigned into the train (156) and test cohorts (100) applying the "caret" package. LASSO regression analysis was employed to select the optimal quantities of genes and corresponding coefficients based on the result from the intersection of univariate Cox regression analysis and K-M method (Figure 3B\&C) in train cohort. Finally, the prognostic signature was constructed on the foundation of five genes that obtained from LASSO regression analysis. The CAFs score of each patient was counted by the following formula: CAFs score = $(0.068607683) *$ XPNPEP $2+(0.07245927) *$ MDM $2+(0.029068022) *$ MFAP5 $+(0.142646307) *$ PAPPA $+(0.09195161) *$ FGF10.

To examine the potential prognostic value of the signature to predict the OS of soft tissue sarcoma samples, the whole cohort were divided into low CAFs score group and high CAFs score group in view of the median value of CAFs score. The K-M curve showed that patients in the low CAFs score group had 
better survival compared to these in the high CAFs score group $(P<0.001)$ (Figure $4 A)$. Besides, we applied the ROC curves to illustrate the precision of the five CAFs-related genes in the prediction of the 1-, 3- and 5- year overall survival of soft tissue sarcoma patients. The 1-, 3- and 5-year survival AUC values were $0.773,0.724$, and 0.726 , respectively (Figure 4B). Additionally, the heatmap, survival score and survival status showed the soft tissue sarcoma samples in the high CAFs score group associated with more deaths, and the expression of these five genes. (Figure $4 \mathrm{C}$ ). All of results were validated in the test and whole cohorts as displayed in Supplementary Figure S2.

Finally, the Cox regression analysis were used to determine if the CAFs score can be considered as an independent prognostic marker of overall survival in soft tissue sarcoma patients. The result of univariate Cox regression analysis demonstrated that the CAFs score $(P<0.001, H R=1.550,95 \% \mathrm{Cl}: 1.263-1.902)$, metastasis $(P=0.002, H R=1.574,95 \% \mathrm{Cl}$ : 1.184-2.091) and histology $(\mathrm{P}=0.041, \mathrm{HR}=0.720,95 \% \mathrm{Cl}$ : 0.526-0.987) were an independent variable for predicting the prognosis of soft tissue sarcoma patients (Figure $5 \mathrm{~A}$ ) in train cohort. The result of multivariate Cox regression analysis also illustrated that the CAFs score $(P<0.001, \mathrm{HR}=1.487,95 \% \mathrm{Cl}: 1.204-1.836)$ and metastasis $(\mathrm{P}=0.005, \mathrm{HR}=1.536,95 \% \mathrm{Cl}$ : 1.141-2.069) were an independent prognostic factor for soft tissue sarcoma patients (Figure 5B) in train cohort. Similarly, CAFs score was an independent prognostic factor for soft tissue sarcoma patients in test and whole cohorts (Supplementary Figure S3). Then, we applied the ROC curve to compare the precision of CAFs score and other clinical features. The AUC of clinical characteristics were lower than that of CAFs score (Figure 5 C). All of results showed the gene signature had a good performance and was an independent prognostic marker for soft tissue sarcoma patients. Based on the constructed CAFsrelated gene model, we established a new nomogram to predict the probability of overall survival of soft tissue sarcoma patients (Figure 5D). And then, the calibration curve displayed that the overall survival rate of 1-year, 3-year and 5-year of the nomogram had a relatively good prediction (Figure 5E).

\section{Chemotherapy response, TMB and cancer stem cell (CSC) of two subgroups patients}

According to the pRRophetic algorithm, we predicted the IC50 of the common chemotherapeutic drugs in GDSC database between two subgroups and discovered that

Most of chemotherapeutic drugs had lower IC50 in the high-risk group, such as JNK.9L, Midostaurin, NVP.TAE684 and WZ.1.84 (Wilcoxon test, $\mathrm{P}<0.01$ ), while some chemotherapeutic drugs, for example, BIRB.0796, JNK inhibitor VII and SL.0101.1 had lower IC50 in the low-risk group (Supplementary Figure S4). These results illustrated that patients in the high-risk group were more sensitive to most of drugs in GDSC database, which may provide some novel chemotherapy drugs to patients in the high-risk subgroup. Besides, to explore the association between the CAFs and TMB, we investigated the TMB of two subgroups. The tumor mutations in two subgroups are displayed in Figure 6A\&B. The results illustrated that mutations in TP53, ATRX, TTN, MUC16 and RB1 were enriched in both subgroups. Besides, missense mutation was the predominant gene alteration type in all these genes except for ATRX, in which nonsense mutation was the most common type. In addition, we analyzed the value of CSC index and risk score to explore the underlying association between risk score and CSC in soft tissue sarcoma. 
We showed the linear correlation between risk score and CSC index in Figure 6C. As displayed in Figure $6 C$, the risk score was negatively associated with CSC index $(R=-0.41, p=2.1 e-07)$, demonstrating that soft tissue sarcoma cells with lower risk score owned more obvious properties of stem cell and a lower rate of cell differentiation.

\section{Analysis of immune status based on CAFs-related risk score}

To explore the immune activity, we employed the sSGSEA to analysis the immune cells infiltration and function between two risk groups in train cohort. The score of ssGSEA computed could demonstrate the infiltration levels of immune cells and immune pathways. The infiltration degree of B cells, CD $8^{+} \mathrm{T}$ cells, DCs, neutrophils, T helper cells, Th1 cells, Th2 cells, regulatory T cell (Treg) and tumor-infiltrating lymphocyte $(\mathrm{TIL})$ were remarkably high in the high CAFs-related risk group $(P<0.05$, Figure 7A). The immune pathway of APC co_stimulation, CCR, check point, cytolytic activity, HLA, inflammation promoting, parainflammation, T cell co-inhibition, T cell co-stimulation, type I IFN Reponse and type II IFN Reponse were also crucially high in high-risk subgroup $(P<0.05$, Figure $7 B)$. Considering that immune check points are significantly different between the two risk subgroups and extremely important for immunotherapies, 47 genes expression of immune check points were examined between two risk subgroups in train cohort. As showed in Figure 7C, 28 immune checkpoints were significantly different expressed and most of them were high expressed in high-risk group.

\section{Discussion}

The tumor microenvironment (TME) is complex and continuously evolving, which comprises cancerassociated fibroblasts, stromal cells, mesenchymal cells, endothelial cells, extracellular matrix and immune cells. Peña et al. revealed that the expression of stanniocalcin-1 (STC1) by CAFs is associated with the metastasis of colorectal cancer ${ }^{16}$. Multiple mechanisms of CAFs can promote cancer progression such as, induction of angiogenesis ${ }^{17}$, immune modulation ${ }^{18}$, recruitment of inflammatory cells $^{19}$ and ECM reshaping ${ }^{20}$. In addition, CAFs originated factors is associated with drug resistance and immune exclusion ${ }^{21}$. Besides, as an intricate mixed system, TME was divided into the immune and nonimmune microenvironment. The components of stromal allocated the non-immune microenvironment ${ }^{22}$, particularly $\mathrm{CAFs}^{23}$. New studies demonstrated the interaction between the stromal and immune components of TIME 24,25 . For example, Mazur et al. revealed that CAFs could enhance the adhesive capability of macrophages and facilitate invasion and metastasis of tumor ${ }^{26}$. Yavuz et al. proved that CAFs derived from breast cancer can enhance the monocytes' differentiation into M2-macrophages ${ }^{27}$.

In this bioinformatics study, we are the first to investigate the reliability of cancer-associated fibroblasts (CAFs) proportion as a biomarker for soft tissue sarcoma prognosis. The soft tissue sarcoma patients were grouped based on the CAFs-related genes identified from the DEGs that between high CAFs score and low CAFs score as well as module of WGCNA. Then, we constructed a polygenic prognostic model based on the CAFs-related genes. To validate the prognostic advantage of CAFs, we assess the 
comprehensive landscapes of tumor microenvironment, tumor genomics, tumor mutation burden and chemosensitivity of CAFs density-stratified soft tissue sarcoma. The CAFs score of each patient on the foundation of the hub genes between through using univariate Cox regression analysis and LASSO regression analysis. We then explored the prognostic capability and biological functions in soft tissue sarcoma. The K-M and ROC curves verified the predictive value of the signature is steady in SARC cohort. Besides, the Cox regression analysis confirmed the five-gene (XPNPEP2, MFAP5, FGF10, MDM2 and PAPPA) signature has independent predictive value for prognosis. And both of five genes were risk factors. Aminopeptidase P2 (XPNPEP2), a proline hydrolytic enzyme, was remarkably overexpressed in cervical cancer and associated with $\mathrm{SiHa}$ and HeLa cells invasion and migration via inducing epithelialmesenchymal transition ${ }^{28}$. Besides, XPNPEP2 was associated with lymph node metastasis and local invasion in prostate cancer patients ${ }^{29}$. Microfibrillar-associated protein 5 (MFAP5), a part of elastic microfibers and an oncogenic protein in several kinds of cancers, is derived from CAFs. Zhou et al. revealed that CAFs-derived MFAP5 is overexpressed in bladder cancer and associated with prognosis as well as promotes cancer proliferation and metastasis by activating NOTCH2/HEY1 signaling pathway ${ }^{30}$. Similarly, stromal fibroblast-derived MFAP5 facilitate cells invasion and migration by regulating the Notch1/slug signaling in breast cancer ${ }^{31}$ and activating of MAPK and AKT pathways in human oral tongue squamous cell carcinoma ${ }^{32}$. Antibody clone $130 \mathrm{~A}$, a MFAP5-targeting therapeutic monoclonal antibody, can suppress pancreatic and ovarian cancer progression, stimulates chemosensitivity and decreases fibrosis ${ }^{33}$. FGF10 is discovered in stromal cells and induced cells migration and invasion via interacting with FGFR2-IIlb in pancreatic cancer ${ }^{34}$. MDM2 enhanced cells invasion and growth via regulating $A K T / m T O R C 1$ signaling in lung cancer ${ }^{35}$. Most of these genes were associated with tumor proliferation and invasion by mediating CAFs in various cancers, but the underlying mechanisms of soft tissue sarcoma were unclear. Therefore, it is necessary to reveal their pathogenic mechanisms and prognosis in soft tissue sarcoma in the further studies.

Besides, we showed the profile of TMB in soft tissue sarcoma patients, demonstrating that TP53, ATRX, TTN, MUC16 and RB1 were the most important mutated genes. TP53, as a well-known tumor suppressor, participate in the occurrence and development of tumor via regulating the cell proliferation, angiogenesis, apoptosis, and DNA repair ${ }^{36}$. Multiple studies have indicated the TP53 is an often-mutated gene in numerous tumors and is associated with prognosis ${ }^{37}$. It illustrated that soft tissue patients with wild-type TP53 tumors had better survival than that of TP53 mutations ${ }^{37}$. The P53 protein is associated with genome stability maintenance as well as tumor suppression, and the study showed that the local activation of P53 in tumor microenvironment could increase the response of antitumor immunity 38 . Besides, high-expressed of mutated TP53 is correlated with resistance to several cytotoxic drugs applied for soft tissue sarcoma patients, for example, doxorubicin (anthracyclines) and gemcitabine (antimetabolites) $^{39}$. The whole-exome sequencing (WES) data of soft tissue sarcoma illustrated ATRX and RB1 are the significantly mutated genes ${ }^{40}$. ATRX encodes a SWI/SNF-like chromatin remodeling protein and often mutates in distinct tumors, such as osteosarcoma, neuroblastoma, and pediatric 
adrenocortical carcinoma ${ }^{41}$. However, the specific pathogenic mechanisms need to be further studied. MUC16 mutations are associated with tumor mutation load and prognosis in gastric cancer patients ${ }^{42}$.

We further assessed the likelihood of response to chemotherapeutic drugs in the two risk subgroups utilizing the pRRophetic algorithm. Notably, most of chemotherapeutic drugs had lower IC50 in the highrisk group, which may provide some novel chemotherapy drugs to patients in the high-risk subgroup.

There are a few limitations in this study. Firstly, there was lack of basic experiment to verify the identified prognostic associated CAFs-related genes' expression in cells/tissues. Secondly, our signature was established based on the public dataset, some important clinical data was not complete and unavailable, which may introduce potential basis or errors. Thirdly, the findings of our study need to be verified in large-scale and prospective studies.

\section{Conclusion}

Our bioinformatics analyses have identified a CAFs gene signature including five CAFs-related genes and constructed the prognostic value in soft tissue sarcoma. Our results extremely revealed the indispensable role of CAFs in soft tissue sarcoma. The conclusion needs further experimental studies for verification.

\section{Abbreviations}

STS soft-tissue sarcomas

CAFs cancer-associated fibroblasts

WGCNA weighted gene co-expression network analysis

TCGA The Cancer Genome Atlas

DEGs differentially expressed genes

ROC receiver-operating characteristic

K-M Kaplan-Meier

TMB Tumor mutation burden

GDSC Genomics of Drug Sensitivity in Cancer

OS Overall survival

\section{Declarations}

\section{Authors' contributions}


Bo Xiao designed the research study; Bo Xiao, Liyan Liu, Zhuoyuan Chen, Yu Xia, Ziyue Zhao and Yi Zeng performed the literature search and statistical analysis; and Bo Xiao, Aoyu Li, Yu Xia, Pingxiao Wang, Ziyue Zhao and Cheng Xiang interpreted the data and drafted the manuscript. Hui Li and Tao Xiao are corresponding authors. Tao Xiao and Hui Li critically revised the manuscript. All authors read and approved the final manuscript.

\section{Ethics approval and consent to participate}

There was no ethics associated with this work because the study purely used the data available in publicly accessible databases.

\section{Conflicts of interest}

All co-authors approved the final version and agreed to the submission.

\section{Availability of data and materials}

The expression profile data and clinical information of this study are publicly available in the TCGA-SARC databases (https://portal.gdc.cancer.gov/). The TMB values of the SARC are publicly available in TCGAmutation (https://portal.gdc.cancer.gov/).

\section{Funding}

This article was funded by including the National Nature Science Foundation of China (grant number 81502332, 82072977), Research Project of Human Health Commission (grant number B2019162), the Nature Science Foundation of Hunan (grant number 2019JJ50861) and the Fundamental Research Funds for the Central Universities of Central South University (grant number 2021zzts0380).

\section{References}

1. Gamboa, A. C., Gronchi, A. \& Cardona, K. Soft-tissue sarcoma in adults: An update on the current state of histiotype-specific management in an era of personalized medicine. CA Cancer J Clin 70, 200-229, doi:10.3322/caac.21605 (2020).

2. Siegel, R. L., Miller, K. D. \& Jemal, A. Cancer statistics, 2019. CA Cancer J Clin 69, 7-34, doi:10.3322/caac.21551 (2019).

3. Zagars, G. K. et al. Prognostic factors for patients with localized soft-tissue sarcoma treated with conservation surgery and radiation therapy: an analysis of 1225 patients. Cancer $97,2530-2543$, doi:10.1002/cncr.11365 (2003).

4. Wang, X., Liu, S., Zhao, X., Fang, E. \& Zhao, X. The value of C-reactive protein as an independent prognostic indicator for disease-specific survival in patients with soft tissue sarcoma: A metaanalysis. PLoS One 14, e0219215, doi:10.1371/journal.pone.0219215 (2019). 
5. Kalluri, R. The biology and function of fibroblasts in cancer. Nat Rev Cancer 16, 582-598, doi:10.1038/nrc.2016.73 (2016).

6. Ziani, L., Chouaib, S. \& Thiery, J. Alteration of the Antitumor Immune Response by Cancer-Associated Fibroblasts. Front Immuno/ 9, 414, doi:10.3389/fimmu.2018.00414 (2018).

7. Sugihara, H. et al. Cancer-associated fibroblast-derived CXCL12 causes tumor progression in adenocarcinoma of the esophagogastric junction. Med Onco/ 32, 618, doi:10.1007/s12032-0150618-7 (2015).

8. Givel, A. M. et al. miR200-regulated CXCL12beta promotes fibroblast heterogeneity and immunosuppression in ovarian cancers. Nat Commun 9, 1056, doi:10.1038/s41467-018-03348-z (2018).

9. Barrett, R. \& Pure, E. Cancer-associated fibroblasts: key determinants of tumor immunity and immunotherapy. Curr Opin Immuno/ 64, 80-87, doi:10.1016/j.coi.2020.03.004 (2020).

10. Mizutani, Y. et al. Meflin-Positive Cancer-Associated Fibroblasts Inhibit Pancreatic Carcinogenesis. Cancer Res 79, 5367-5381, doi:10.1158/0008-5472.CAN-19-0454 (2019).

11. Qin, X. et al. Cancer-associated Fibroblast-derived IL-6 Promotes Head and Neck Cancer Progression via the Osteopontin-NF-kappa B Signaling Pathway. Theranostics 8, 921-940, doi:10.7150/thno.22182 (2018).

12. Li, T. et al. TIMER: A Web Server for Comprehensive Analysis of Tumor-Infiltrating Immune Cells. Cancer Res 77, e108-e110, doi:10.1158/0008-5472.CAN-17-0307 (2017).

13. Schumacher, T. N., Kesmir, C. \& van Buuren, M. M. Biomarkers in cancer immunotherapy. Cancer Cell 27, 12-14, doi:10.1016/j.ccell.2014.12.004 (2015).

14. Lv, J., Zhu, Y., Ji, A., Zhang, Q. \& Liao, G. Mining TCGA database for tumor mutation burden and their clinical significance in bladder cancer. Biosci Rep 40, doi:10.1042/BSR20194337 (2020).

15. Geeleher, P., Cox, N. \& Huang, R. S. pRRophetic: an R package for prediction of clinical chemotherapeutic response from tumor gene expression levels. PLoS One 9, e107468, doi:10.1371/journal.pone.0107468 (2014).

16. Pena, C. et al. STC1 expression by cancer-associated fibroblasts drives metastasis of colorectal cancer. Cancer Res 73, 1287-1297, doi:10.1158/0008-5472.CAN-12-1875 (2013).

17. Bergers, G. \& Benjamin, L. E. Tumorigenesis and the angiogenic switch. Nat Rev Cancer 3, 401-410, doi:10.1038/nrc1093 (2003).

18. Mhaidly, R. \& Mechta-Grigoriou, F. Fibroblast heterogeneity in tumor micro-environment: Role in immunosuppression and new therapies. Semin Immunol 48, 101417, doi:10.1016/j.smim.2020.101417 (2020).

19. Coussens, L. M. \& Werb, Z. Inflammation and cancer. Nature 420, 860-867, doi:10.1038/nature01322 (2002).

20. Theret, N. et al. Increased extracellular matrix remodeling is associated with tumor progression in human hepatocellular carcinomas. Hepatology 34, 82-88, doi:10.1053/jhep.2001.25758 (2001). 
21. Biffi, G. \& Tuveson, D. A. Diversity and Biology of Cancer-Associated Fibroblasts. Physiol Rev 101, 147-176, doi:10.1152/physrev.00048.2019 (2021).

22. Roma-Rodrigues, C., Mendes, R., Baptista, P. V. \& Fernandes, A. R. Targeting Tumor Microenvironment for Cancer Therapy. Int J Mol Sci 20, doi:10.3390/ijms20040840 (2019).

23. Zeltz, C. et al. Cancer-associated fibroblasts in desmoplastic tumors: emerging role of integrins. Semin Cancer Biol 62, 166-181, doi:10.1016/j.semcancer.2019.08.004 (2020).

24. Chen, D. et al. Long Non-coding RNAs as Communicators and Mediators Between the Tumor Microenvironment and Cancer Cells. Front Onco/ 9, 739, doi:10.3389/fonc.2019.00739 (2019).

25. Xu, S. J., Hu, H. T., Li, H. L. \& Chang, S. The Role of miRNAs in Immune Cell Development, Immune Cell Activation, and Tumor Immunity: With a Focus on Macrophages and Natural Killer Cells. Cells 8 , doi:10.3390/cells8101140 (2019).

26. Mazur, A., Holthoff, E., Vadali, S., Kelly, T. \& Post, S. R. Cleavage of Type I Collagen by Fibroblast Activation Protein-alpha Enhances Class A Scavenger Receptor Mediated Macrophage Adhesion. PLoS One 11, e0150287, doi:10.1371/journal.pone.0150287 (2016).

27. Gok Yavuz, B. et al. Cancer associated fibroblasts sculpt tumour microenvironment by recruiting monocytes and inducing immunosuppressive PD-1(+) TAMs. Sci Rep 9, 3172, doi:10.1038/s41598019-39553-z (2019).

28. Cheng, T. et al. XPNPEP2 is overexpressed in cervical cancer and promotes cervical cancer metastasis. Tumour Bio/39, 1010428317717122, doi:10.1177/1010428317717122 (2017).

29. Li, F. et al. XPNPEP2 is associated with lymph node metastasis in prostate cancer patients. Sci Rep 9 , 10078, doi:10.1038/s41598-019-45245-5 (2019).

30. Zhou, Z. et al. CAFs-derived MFAP5 promotes bladder cancer malignant behavior through NOTCH2/HEY1 signaling. FASEB J 34, 7970-7988, doi:10.1096/fj.201902659R (2020).

31. Chen, Z., Yan, X., Li, K., Ling, Y. \& Kang, H. Stromal fibroblast-derived MFAP5 promotes the invasion and migration of breast cancer cells via Notch1/slug signaling. Clin Trans/ Oncol22, 522-531, doi:10.1007/s12094-019-02156-1 (2020).

32. Principe, S. et al. Proteomic Analysis of Cancer-Associated Fibroblasts Reveals a Paracrine Role for MFAP5 in Human Oral Tongue Squamous Cell Carcinoma. J Proteome Res 17, 2045-2059, doi:10.1021/acs.jproteome.7b00925 (2018).

33. Yeung, T. L. et al. Anticancer Immunotherapy by MFAP5 Blockade Inhibits Fibrosis and Enhances Chemosensitivity in Ovarian and Pancreatic Cancer. Clin Cancer Res 25, 6417-6428, doi:10.1158/1078-0432.CCR-19-0187 (2019).

34. Huijts, P. E. et al. Allele-specific regulation of FGFR2 expression is cell type-dependent and may increase breast cancer risk through a paracrine stimulus involving FGF10. Breast Cancer Res 13, R72, doi:10.1186/bcr2917 (2011).

35. Kamer, I. et al. Stromal-MDM2 Promotes Lung Cancer Cell Invasion through Tumor-Host Feedback Signaling. Mol Cancer Res 18, 926-937, doi:10.1158/1541-7786.MCR-19-0395 (2020). 
36. Fischer, M., Grossmann, P., Padi, M. \& DeCaprio, J. A. Integration of TP53, DREAM, MMB-FOXM1 and RB-E2F target gene analyses identifies cell cycle gene regulatory networks. Nucleic Acids Res 44, 6070-6086, doi:10.1093/nar/gkw523 (2016).

37. Ohnstad, H. O. et al. Correlation of TP53 and MDM2 genotypes with response to therapy in sarcoma. Cancer 119, 1013-1022, doi:10.1002/cncr.27837 (2013).

38. Guo, G., Yu, M., Xiao, W., Celis, E. \& Cui, Y. Local Activation of p53 in the Tumor Microenvironment Overcomes Immune Suppression and Enhances Antitumor Immunity. Cancer Res 77, 2292-2305, doi:10.1158/0008-5472.CAN-16-2832 (2017).

39. Robles, A. I. \& Harris, C. C. Clinical outcomes and correlates of TP53 mutations and cancer. Cold Spring Harb Perspect Bio/ 2, a001016, doi:10.1101/cshperspect.a001016 (2010).

40. Cancer Genome Atlas Research Network. Electronic address, e. d. s. c. \& Cancer Genome Atlas Research, N. Comprehensive and Integrated Genomic Characterization of Adult Soft Tissue Sarcomas. Cell 171, 950-965 e928, doi:10.1016/j.cell.2017.10.014 (2017).

41. Dyer, M. A., Qadeer, Z. A., Valle-Garcia, D. \& Bernstein, E. ATRX and DAXX: Mechanisms and Mutations. Cold Spring Harb Perspect Med 7, doi:10.1101/cshperspect.a026567 (2017).

42. Li, X., Pasche, B., Zhang, W. \& Chen, K. Association of MUC16 Mutation With Tumor Mutation Load and Outcomes in Patients With Gastric Cancer. JAMA Oncol 4, 1691-1698, doi:10.1001/jamaoncol.2018.2805 (2018).

\section{Figures}




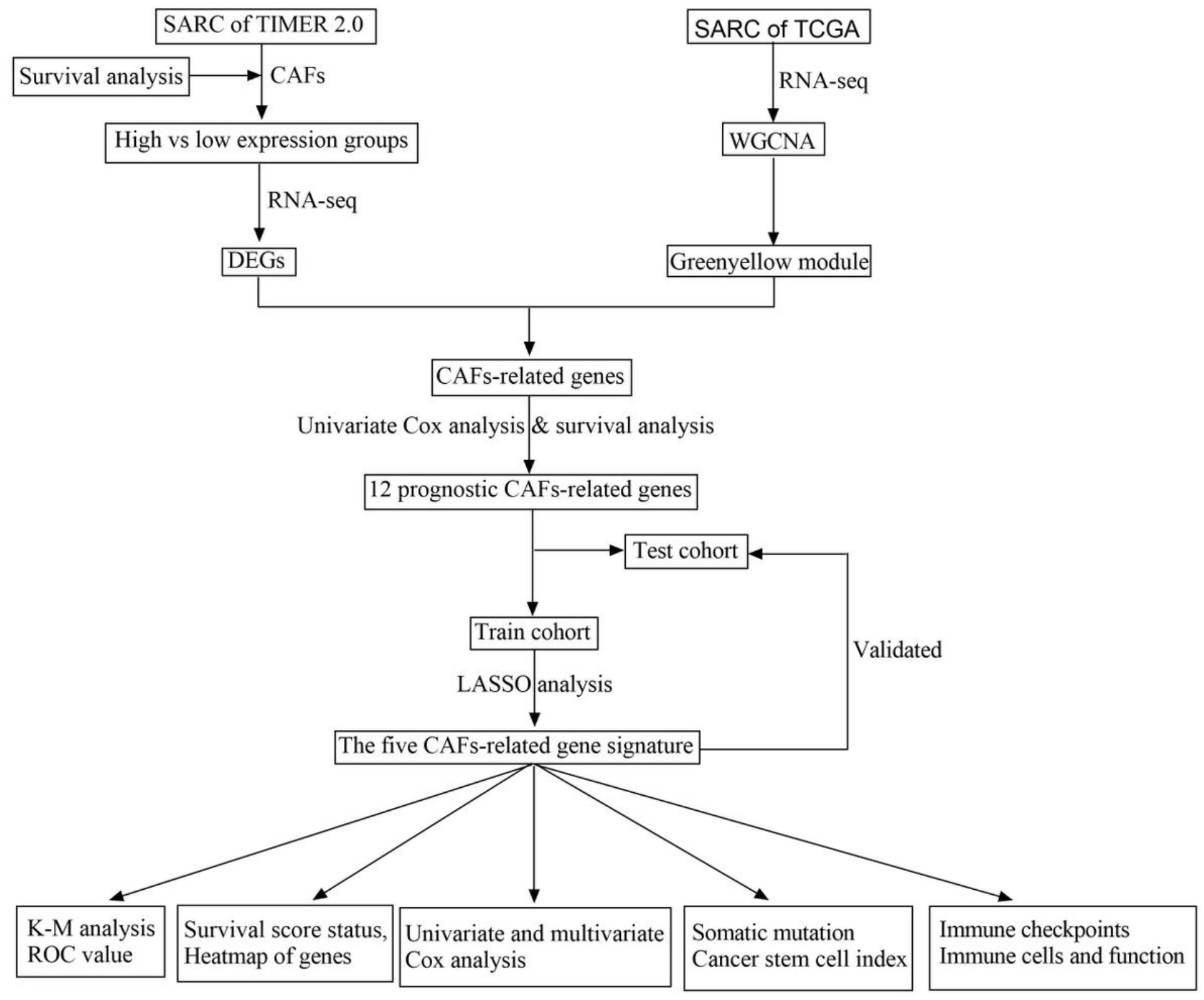

\section{Figure 1}

Flow chart of data gathering and analysis. 
A
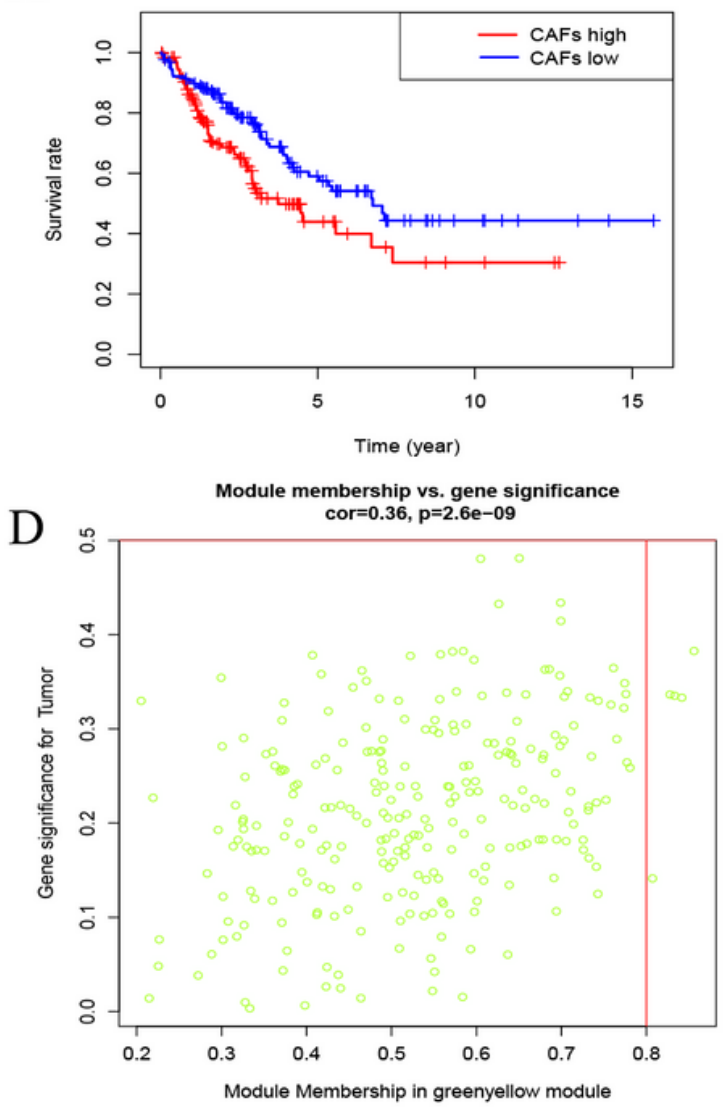

E WGCNA

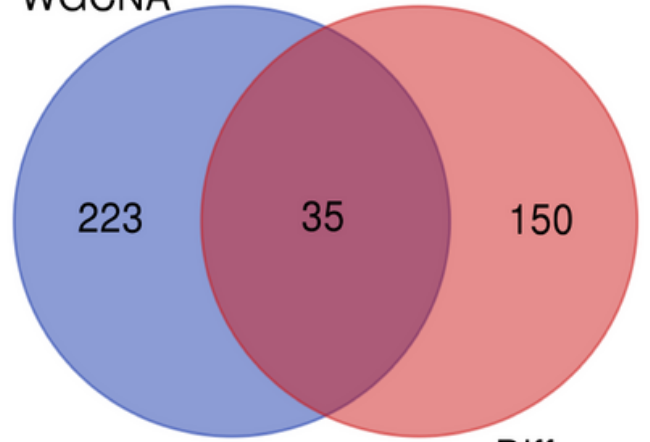

Diff

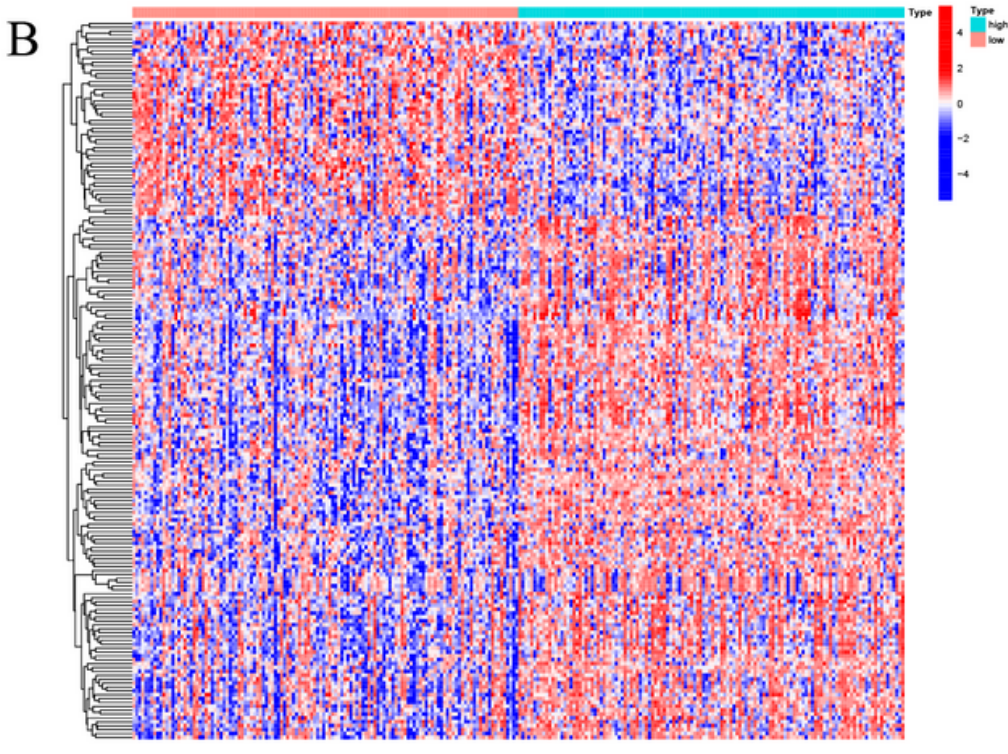

Module-trait relationships

$\mathrm{C}$

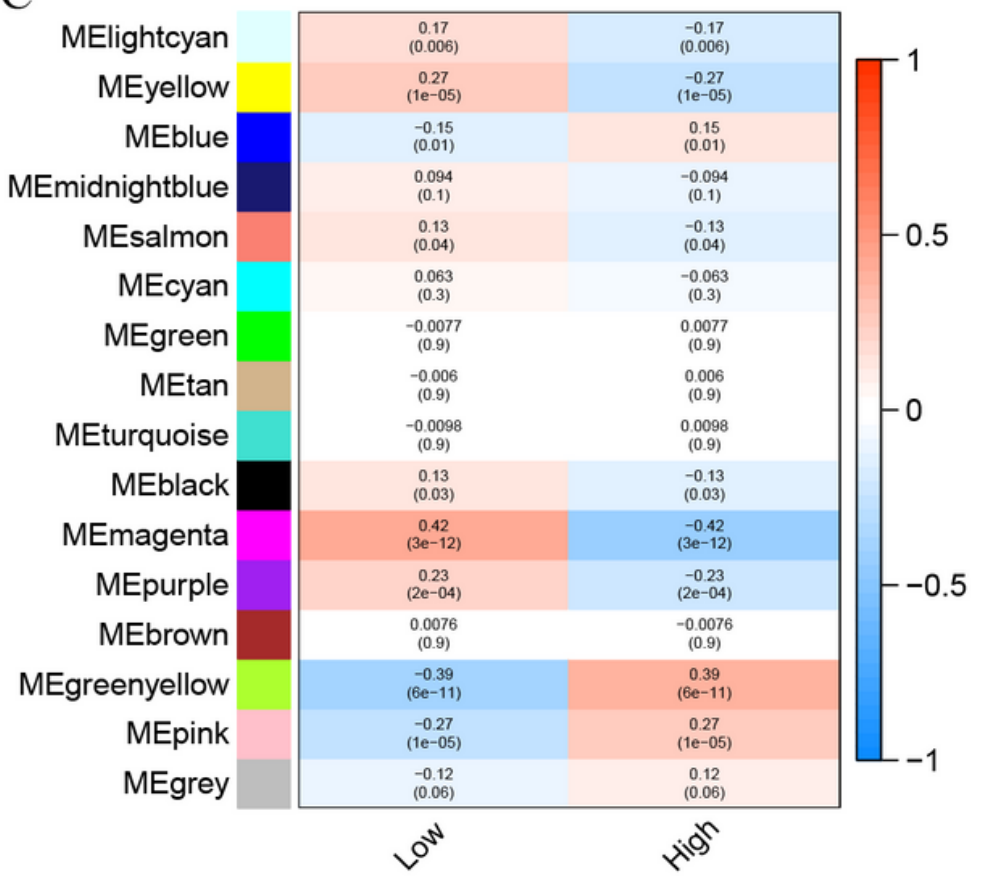

Figure 2

Analysis and selecting out CAFs-related genes. A: K-M curve revealed that patients with high abundance of CAFs had obviously lower OS ( $p=0.014)$; $B$ : The DEGs between high and low CAFs in TCGA-SARC cohort; $\mathrm{C}$ : the correlation analysis between modules and CAFs; $\mathrm{D}$ : The scatterplot illustrates the association between CAFs and intramodular connectivity; $\mathrm{E}$ : The intersection of CAFs-related hub genes between WGCNA and DEGs. 
A

$\begin{array}{lrr} & \text { pvalue } & \text { Hazard ratio } \\ \text { XPNPEP2 } & <.001 & 1.205(1.095-1.325) \\ \text { ADGRD1 } & 0.001 & 1.224(1.081-1.386) \\ \text { SVEP1 } & 0.019 & 1.189(1.028-1.374) \\ \text { TCF21 } & 0.023 & 1.144(1.018-1.286) \\ \text { AVIL } & 0.002 & 1.312(1.108-1.553) \\ \text { ELN } & 0.011 & 1.130(1.028-1.242) \\ \text { SCARA5 } & 0.007 & 1.110(1.029-1.198) \\ \text { SLC35E3 } & 0.003 & 1.276(1.088-1.497) \\ \text { VEGFD } & 0.010 & 1.127(1.028-1.235) \\ \text { MDM2 } & <0.001 & 1.216(1.090-1.357) \\ \text { ADH1C } & 0.025 & 1.124(1.015-1.244) \\ \text { ADAMTS15 } & 0.033 & 1.198(1.015-1.414) \\ \text { PLA2G2A } & 0.008 & 1.094(1.023-1.169) \\ \text { TNXB } & 0.001 & 1.157(1.061-1.263) \\ \text { AIF1L } & 0.033 & 1.132(1.010-1.268) \\ \text { MFAP5 } & 0.003 & 1.119(1.039-1.205) \\ \text { CLEC3B } & 0.005 & 1.127(1.036-1.225) \\ \text { PAPPA } & <0.001 & 1.488(1.221-1.814) \\ \text { FGF10 } & 0.003 & 1.235(1.073-1.422) \\ \text { ABCA9 } & 0.020 & 1.179(1.027-1.354)\end{array}$

B

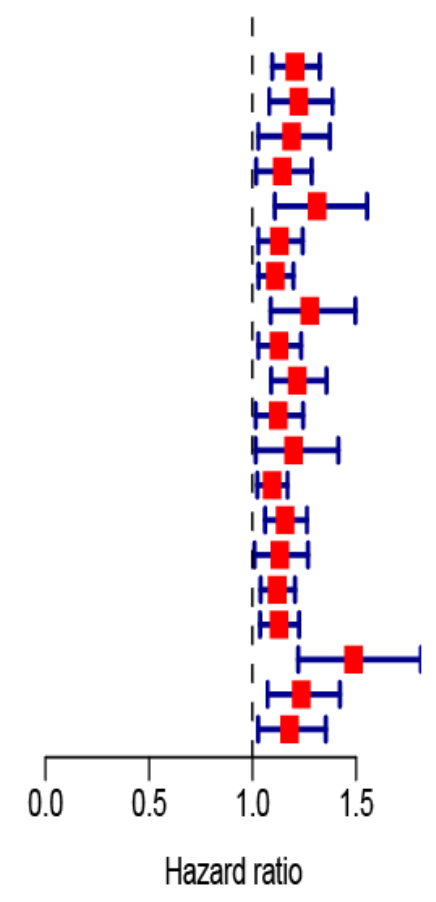

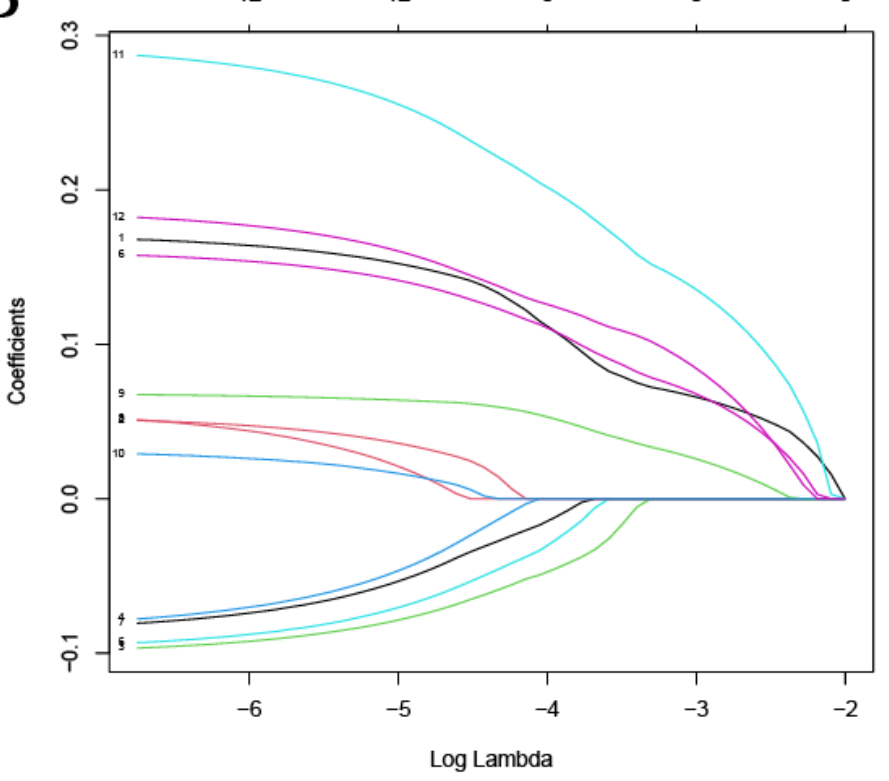

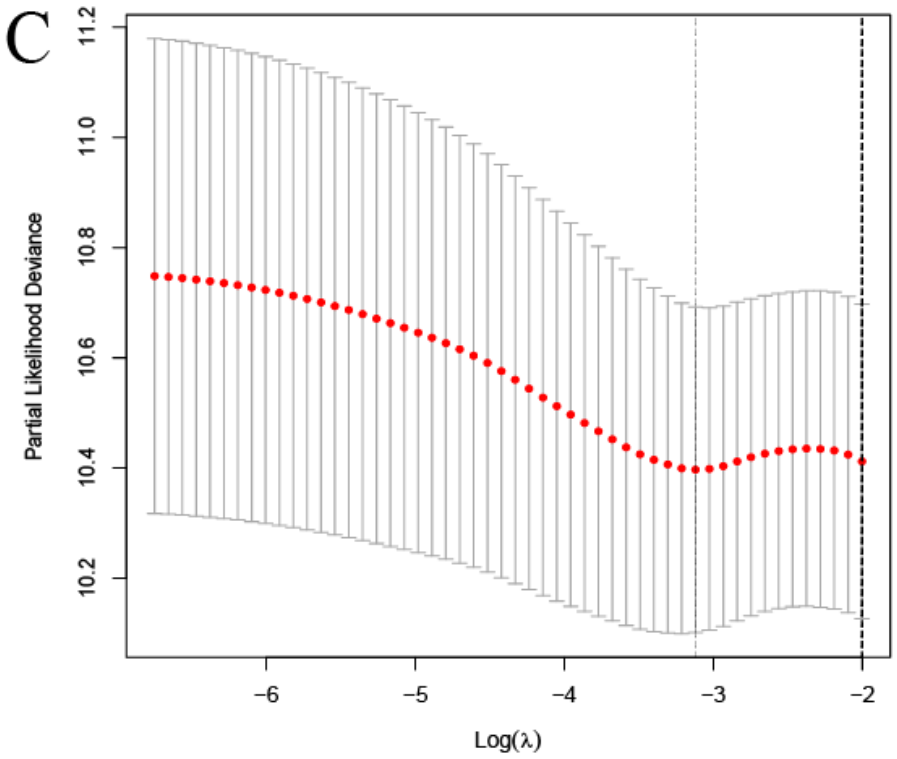

\section{Figure 3}

Construction of CAFs-related gene prognostic signature. The result of univariate Cox regression analysis (A); The results of LASSO Cox analysis (B\&C). 
A
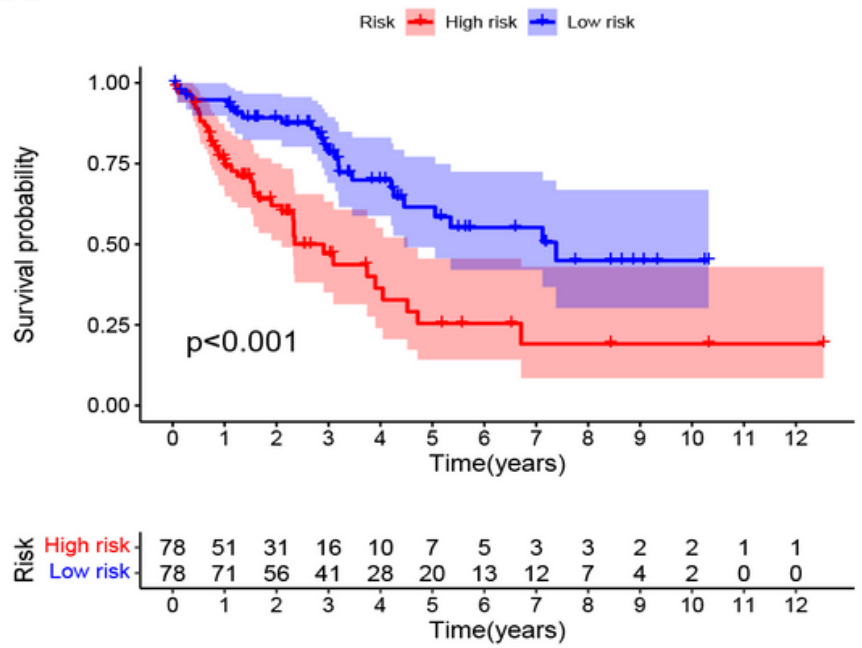

$\mathrm{B}$

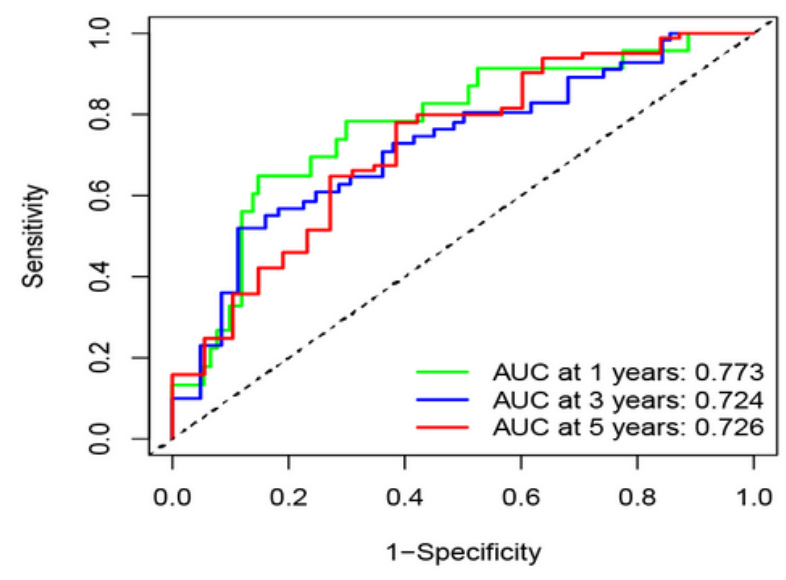

$\mathrm{C}$
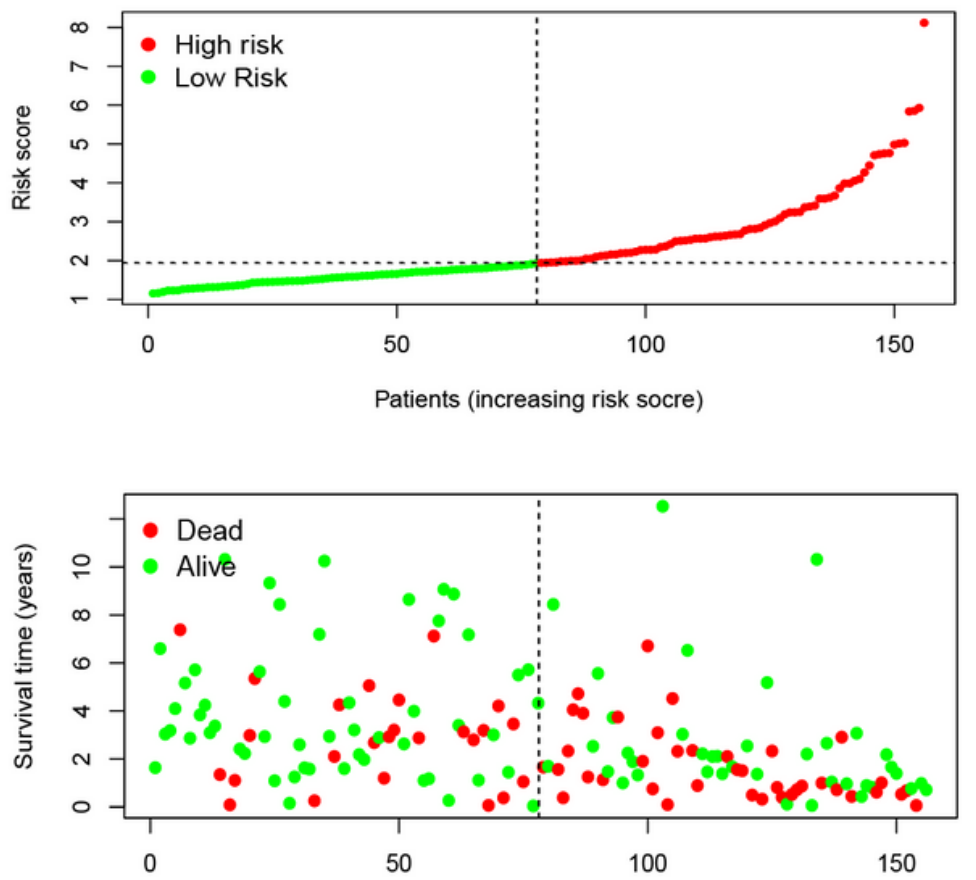

Patients (increasing risk socre)

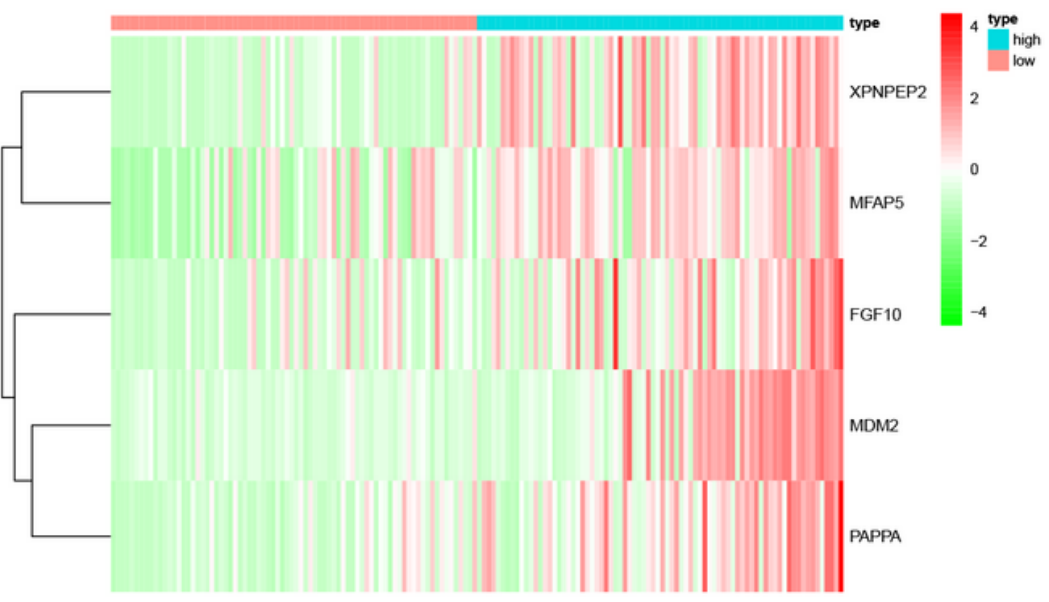

\section{Figure 4}

K-M curve (A), ROC curve (B) and the risk score, survival status as well as mRNA expression (C) of five CAFs-related gene signature. 


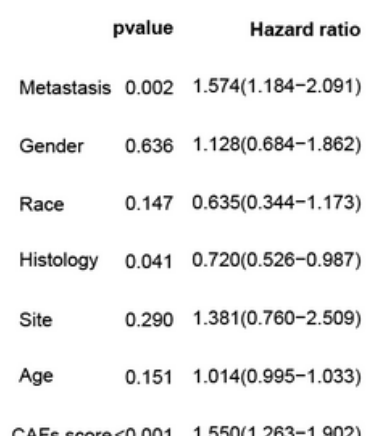

CAFs score $<0.001 \quad 1.550(1.263-1.902)$

\section{$\mathrm{C}$}

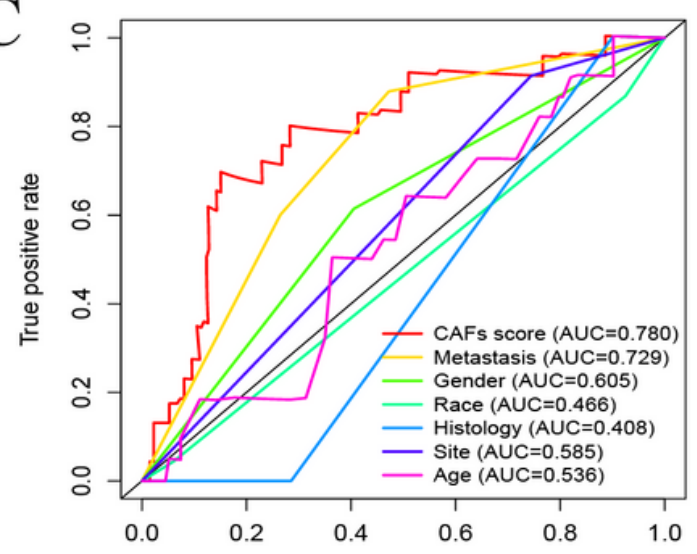

$\mathrm{E}$

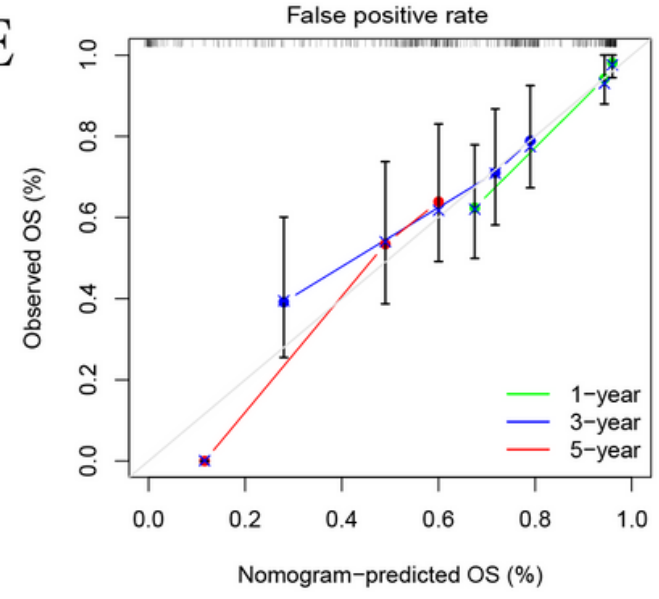

B
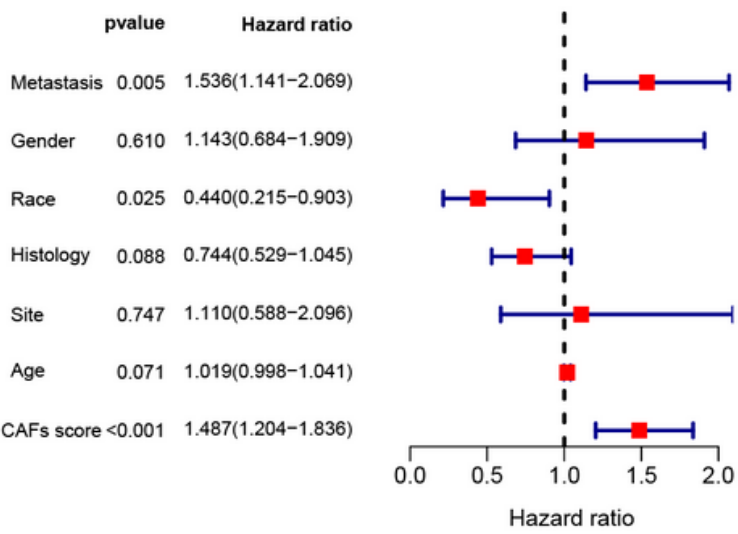

$\mathrm{D}$

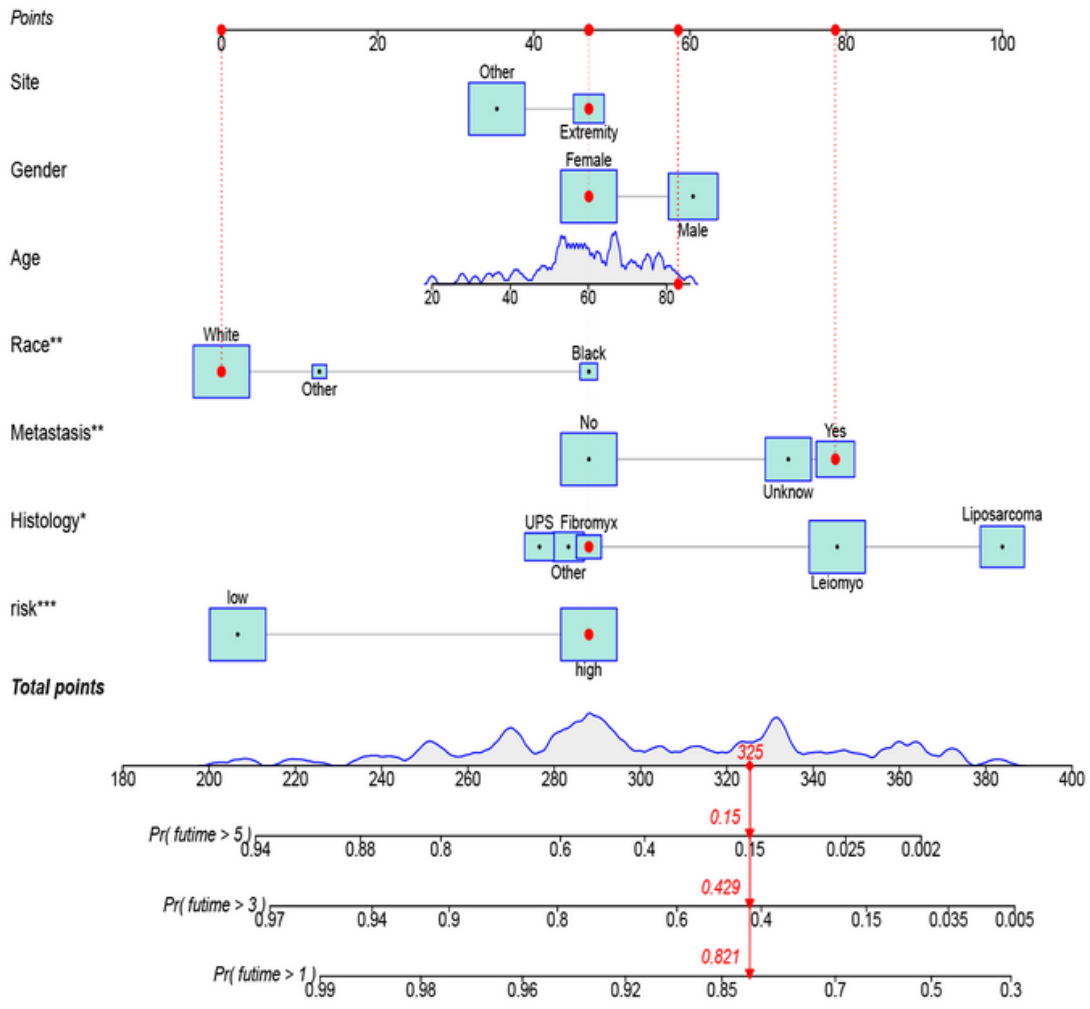

Figure 5

The results of univariate (A), multivariate (B) Cox regression analysis and ROC curve (C) of CAFs score and other clinical features; Nomogram for predicting 1-, 3- and 5-years survival rate of soft tissue sarcoma patients (D); Calibration curve to predict OS rate of soft tissue sarcoma patients (E). 


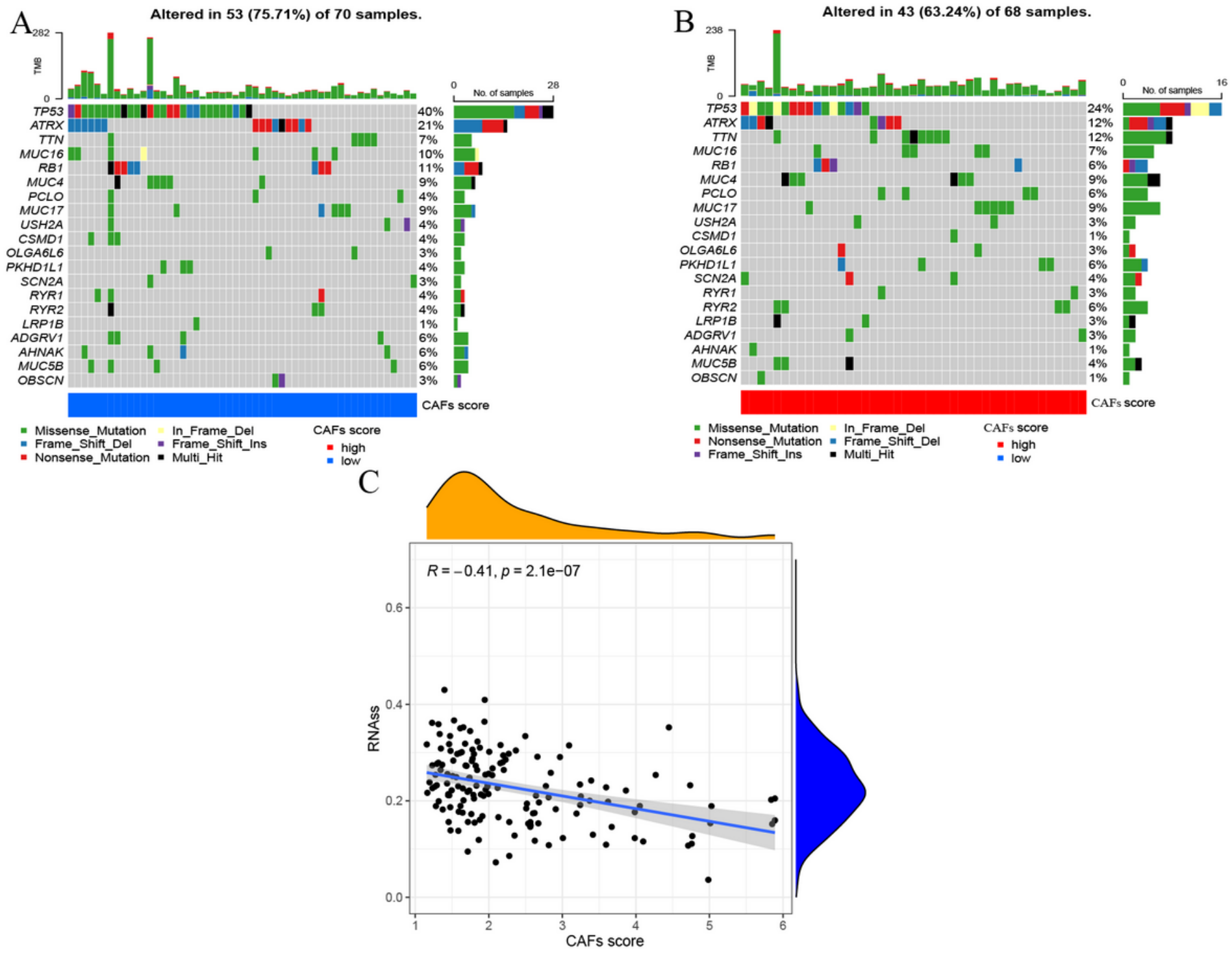

Figure 6

The waterfall plots of most frequently altered genes between two risk subgroups (A\&B); Correlation analysis between CAFs score and RNAss (C). 
A

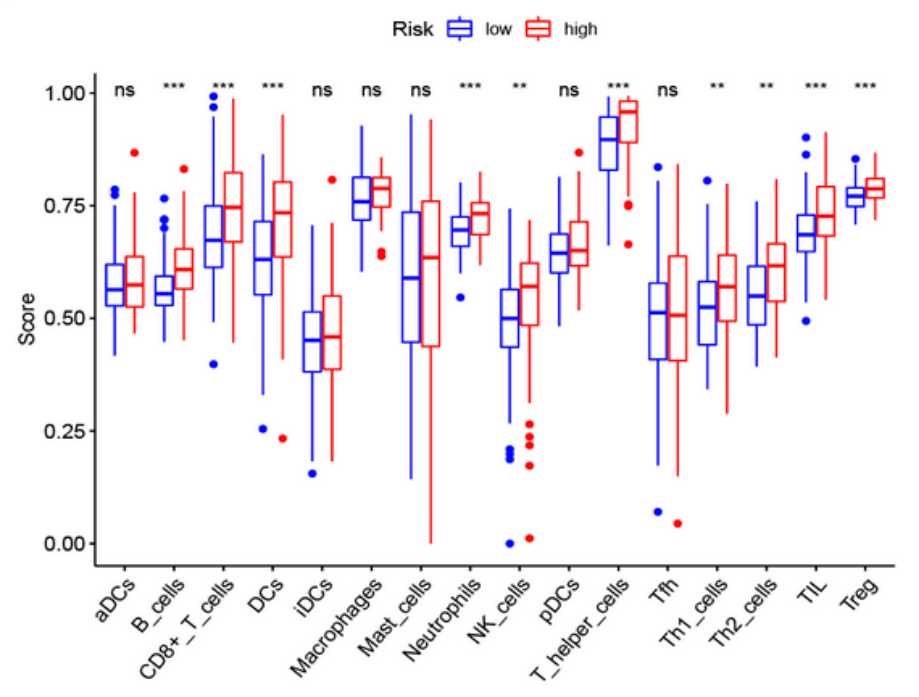

$\mathrm{C}$
$\mathrm{B}$

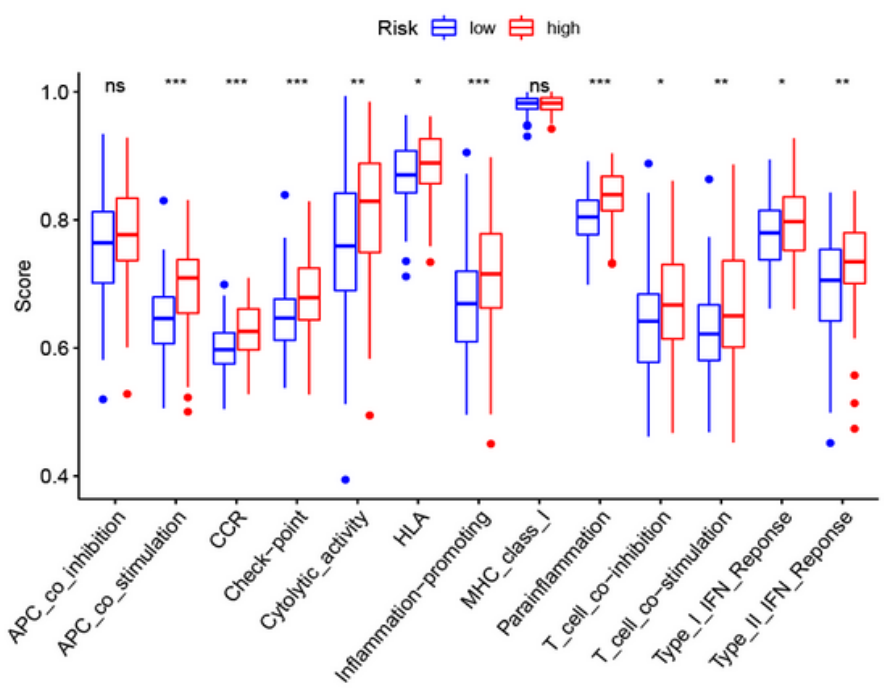

Risk 追 low 官 high

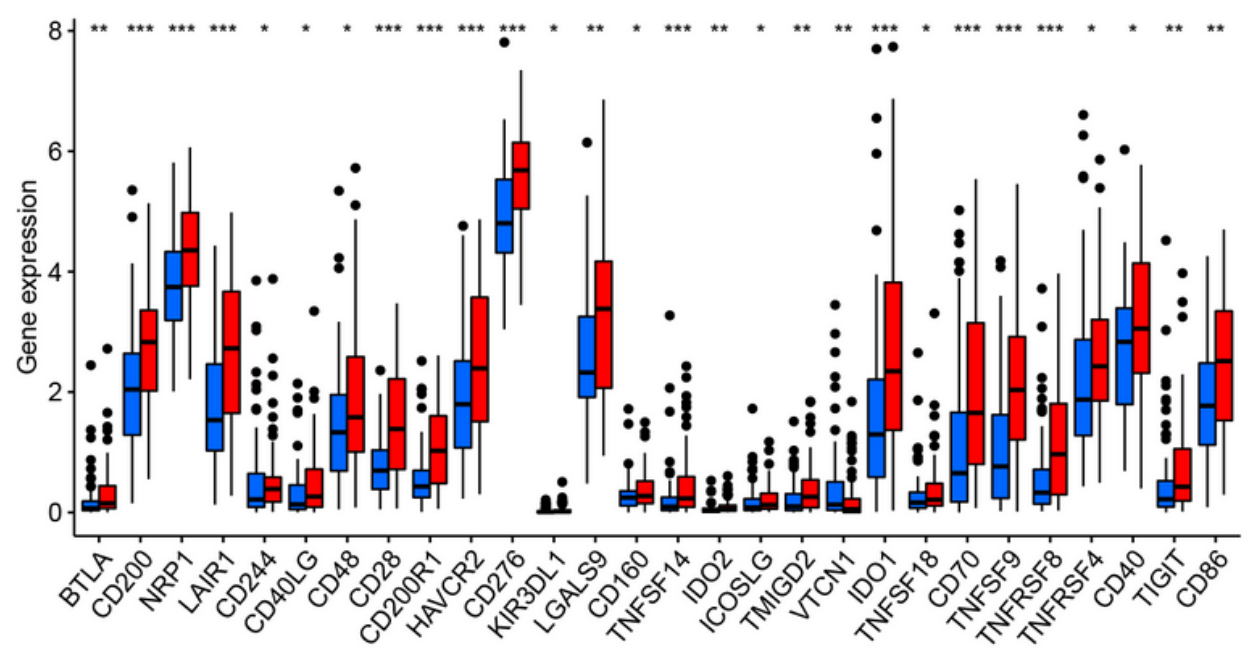

Figure 7

Analysis of immune status and immune checkpoints based on CAFs score. Comparisons of immune cells (A), immune functions (B) and immune checkpoints (C) between distinct risk subgroups. $\left({ }^{\star} p<0.05\right.$, ${ }^{\star \star} p<$ $\left.0.01,{ }^{* * *} p<0.001\right)$.

\section{Supplementary Files}

This is a list of supplementary files associated with this preprint. Click to download.

- Supplementaryfigureslegends.docx

- supplementaryFigureS2.tif

- supplementaryFigureS3.tif

- supplementaryFigureS4.tif 
- supplementaryfigureS1.tif 